\title{
Synchrotron fluence of nonlinearly cooled relativistic electrons with instantaneous flat power law injection (Research Note)
}

\author{
M. Zacharias and R. Schlickeiser
}

\begin{abstract}
Institut für Theoretische Physik, Lehrstuhl IV: Weltraum- und Astrophysik, Ruhr-Universität Bochum, 44780 Bochum, Germany e-mail: rsch@tp4.rub.de
\end{abstract}

Received 19 September 2008 / Accepted 5 February 2009

\begin{abstract}
Context. In powerful cosmic nonthermal radiation sources with a dominant magnetic field self generation, partition between the energy densities in magnetic fields and relativistic electrons is expected. As the electrons cool by synchrotron emission, the magnetic field strength becomes time-dependent and adjusts itself to the actual kinetic energy density of the radiating electrons.

Aims. For the case of instantaneous injection of power law distributed electrons with a flat power law spectral index, the nonlinear synchrotron fluence is calculated.

Methods.

Results. For flat (spectral index $1<s<2$ ) injected power laws the nonlinear synchrotron fluence at small frequencies approaches a power law $\propto v^{-0.6}$, independent of the value of $s$, which is identical to the synchrotron fluence behaviour for steep injected power laws.
\end{abstract}

Key words. radiation mechanisms: non-thermal - galaxies: active - ISM: cosmic rays

\section{Introduction}

The field of AGN multiwavelength studies is entering an exciting new phase. The new generation of ground-based air Cherenkov telescopes such as HESS and MAGIC have detected more than 20 AGNs as powerful variable high-energy photon emitters: with the noteworthy exception of M 87 all of them belong to the blazar class which is characterized by rapid time variability at all wavelengths and often have established superluminal motion components at radio and $\mathrm{mm}$ frequencies. Their spectral energy distributions are characterized by two broad peaks, one in the optical-UV-X-ray band and one in the GeV to $\mathrm{TeV}$ region. The recently launched FERMI/GLAST-satellite mission will close the current observational gap at $\mathrm{MeV}-\mathrm{GeV}$ energies. Together with the high-time resolution of flaring blazar emission (Aharonian et al. 2007), observational material of unprecedented detail and accuracy is available that allows us to perform crucial tests of our current understanding of the physical processes responsible for the generation of radiation in these sources.

The broadband continuum spectra of blazars are dominated by nonthermal emission and often consist of two distinct broad components. From $\gamma-\gamma$-opacity arguments (Schlickeiser 1996) the emission is considered to originate in relativistically moving jet components outside of the $\gamma-\gamma$-photosphere. While there is considerable debate concerning the nature of the high energy spectral component (decay of neutral pions in hadronic emission models versus inverse Compton scattering of intrinsic (SSC) and external low-energy photons in leptonic emission models), it is agreed that the low-energy component is synchrotron radiation from highly relativistic electrons. This requires the presence of strong enough tangled magnetic fields at rather large distances from the central object in order to magnetically confine the radiating particles to the emission knots and to produce synchrotron radiation at ultraviolet and even X-ray frequencies. Because the magnetic fields anchored in the accretion disk and/or the central supermassive black hole quickly decrease with increasing distance, it has been proposed that the outflowing emission blobs self generate these strong magnetic fields by kinetic plasma instabilities in their interaction with the ambient partially ionized interstellar or intergalactic medium (Stockem et al. 2007). Such magnetic microturbulence is also essential for gas flow entropisation to form shock wave structures in collision-poor plasma environments. It has been demonstrated that relativistic counterstreams indeed generate magnetic field fluctuations at the subequipartition level differently in hadronic and leptonic counterstreams. In the absence or at low values of a finite guide magnetic field, the generated aperiodic transverse magnetic fluctuations from the filamentation instability grow quicker than longitudinal oscillations in the relativistic flow. Aperiodic magnetic field fluctuations pitch-angle scatter inflowing particles and build up to equipartition strength with the kinetic energy of the captured particles. These fluctuations are able to confine relativistic charged particles by rapid pitch-angle scatterings.

Schlickeiser \& Lerche (2007) have argued that under such conditions the generated magnetic fields are closely related to the processes energising ultra-high energy radiating electrons in these sources. Then the magnetic field strength becomes timedependent and adjusts itself to the actual kinetic energy density of the radiating electrons. This implies a new rapid nonlinear cooling process of relativistic electrons. Under such partition conditions the synchrotron cooling of the relativistic electrons depends on the kinetic energy density of the radiating electrons 
that is an integral over the differential spectral density. As a consequence, the evolution of the relativistic electron energy spectra exhibits nonlinear behaviour because the cooling of an individual particle is stronger with a higher kinetic energy density of the whole charged particle population. As a consequence, the temporal behaviour of the different photon emissivities will also be influenced allowing a verification of the presence of nonlinear cooling processes in these sources.

Schlickeiser \& Lerche (2008, hereafter referred to as SL) showed that the behaviour of the synchrotron fluence of relativistic electrons depends strongly on the injected energy spectrum of the electrons in the magnetic field. If relativistic electrons are injected instantaneously at time $t=0$ at a rate $q_{0}$ with a steep (i.e. spectral index $s>2)$ power-law $\left(\propto \gamma^{-s}, \gamma_{1} \leq\right.$ $\gamma \leq \gamma_{2}$ ) dependence on the electron Lorentz factor $\gamma$ between a lower $\left(\gamma_{1}\right)$ and an upper $\left(\gamma_{2}=g_{2} \gamma_{1}\right)$ cut-off, the synchrotron fluence $F(f)$ becomes a broken power-law in the (normalized) frequency $f=v / K_{0} U_{0}^{1 / 2} \gamma_{1}^{2}$, where $U_{0}=q_{0} /(s-2) \gamma_{1}^{s-2}$ and $K_{0}=3 c \sqrt{e_{B} r_{0} / 2 \pi}=1.9 \times 10^{4} \mathrm{~Hz} \mathrm{~cm}^{3 / 2}$ :

$$
\begin{aligned}
& F(f \geq 1)=\frac{N_{0} q_{0}}{A_{0} \gamma_{1}^{s-1} U_{0}^{\frac{1}{2}}(s-1)} f^{-\frac{s}{2}}\left[1-\left(\frac{f}{g_{2}^{2}}\right)^{\frac{s-1}{2}}\right] \\
& F(f \ll 1 / 4)=\left(\frac{5}{4}\right)^{s-2} \frac{N_{0} q_{0}\left(1-g_{2}^{-1}\right)}{A_{0} \gamma_{1}^{s} U_{0}^{\frac{1}{2}}} \times\left(\frac{s-2}{s-1}\right)^{-\frac{1}{5}} f^{-\frac{3}{5}} .
\end{aligned}
$$

As in SL the constants are $A_{0}=4 c \sigma_{T} e_{B} / 3$ and $N_{0}=$ $L m_{e} c^{3} \sigma_{T} e_{B} /\left(6 \pi K_{0}\right)$.

It is the purpose of this research note to investigate the synchrotron fluence for the case of the instantaneous injection of a flat (i.e. spectral index $1<s<2$ ) power-law of electrons because several acceleration models predict flat power laws of accelerated particles due to modified scattering center compression ratios at cosmic shock waves (Vainio \& Schlickeiser 1999; Tammi \& Vainio 2005) and/or the dominant operation of downstream 2nd-order Fermi acceleration (Virtanen et al. 2003; Virtanen \& Vainio 2005; Stawarz \& Petrosian 2008). Our approach follows closely the steps in SL.

\section{Analytical calculation of the fluence}

We start from Eq. (SL-80) for the optically thin synchrotron radiation intensity from nonlinearly cooling relativistic electrons which with $U_{1}=-U_{0}=q_{0} /(2-s) \gamma_{1}^{s-2}, y=A_{0} t$ and $f=v / K_{0} U_{1}^{1 / 2} \gamma_{1}^{2}$ reads

$$
\begin{aligned}
I_{\mathrm{NL}}(f, y)= & N_{0} \gamma_{1}^{1-s} U^{1 / 2}(y)\left[\frac{U_{1}}{U(y)}\right]^{\frac{1-s}{4}} f^{\frac{1-s}{2}} \\
& \times\left[1-f^{1 / 2}\left(\frac{U_{1}}{U(y)}\right)^{1 / 4} \gamma_{1} T\right]^{s-2} \\
& \times H\left[f-\left(\frac{U_{1}}{U(y)}\right)^{1 / 2} \frac{1}{\left(1+\gamma_{1} T\right)^{2}}\right] \\
& \times H\left[\left(\frac{U_{1}}{U(y)}\right)^{1 / 2} \frac{\gamma_{2}}{\left(1+\gamma_{2} T\right)^{2}}-f\right]
\end{aligned}
$$

with the implicit time variable $T(y)$ defined by

$$
U(y)=\frac{\mathrm{d} T}{\mathrm{~d} y}=q_{0} T^{s-2} \int_{\left(\gamma_{2} T\right)^{-1}}^{\left(\gamma_{1} T\right)^{-1}} \mathrm{~d} v \frac{v^{s-2}}{v+1} .
$$

Substituting $x=\gamma_{1} T$ and using $g_{2}=\gamma_{2} / \gamma_{1}$ we derive $U(x) / U_{1}=$ $J(x)$ with

$J(x)=(2-s) \int_{g_{2}^{-1}}^{1} \mathrm{~d} u \frac{u^{s-2}}{u+x}$

This integral (5) is well approximated by

$J(x)= \begin{cases}g_{2}^{2-s}-1 & \text { for } 0 \leq x \leq g_{2}^{-1} \\ \frac{x^{s-2}}{s-1}-1-\frac{2-s}{s-1} \frac{g_{2}^{1-s}}{x} & \text { for } g_{2}^{-1} \leq x \leq 1 \\ \frac{2-s}{(s-1) x}\left[1-g_{2}^{1-s}\right] & \text { for } x \geq 1\end{cases}$

The fluence is given by the time-integrated synchrotron intensity as

$$
\begin{aligned}
F_{\mathrm{NL}}(f)= & \frac{1}{A_{0}} \int_{0}^{\infty} \mathrm{d} y I_{\mathrm{NL}}(f, y)=\frac{1}{A_{0} \gamma_{1} U_{1}} \int_{0}^{\infty} \mathrm{d} x \frac{I_{\mathrm{NL}}(f, x)}{J(x)} \\
= & F_{0} f^{\frac{1-s}{2}} \int_{0}^{\infty} \mathrm{d} x J^{\frac{s-3}{4}}(x)\left[1-\frac{f^{1 / 2} x}{J^{1 / 4}(x)}\right]^{s-2} \\
& \times H\left[f-\frac{J^{1 / 2}(x)}{(1+x)^{2}}\right] H\left[\frac{J^{1 / 2}(x)}{\left(g_{2}^{-1}+x\right)^{2}}-f\right]
\end{aligned}
$$

with $F_{0}=\left(N_{0} / A_{0} U_{1}^{1 / 2} \gamma_{1}^{s}\right)$. Inserting for $g_{2} \gg 1$ the leading terms of the approximation (6) we find that the fluence is determined by the sum of three terms:

$$
F_{\mathrm{NL}}(f)=F_{0} f^{\frac{1-s}{2}}\left[F_{1}(f)+F_{2}(f)+F_{3}(f)\right]
$$

with

$$
\begin{aligned}
F_{1}(f)= & \frac{\left[g_{2}^{2-s}-1\right]^{\frac{s-2}{4}}}{(s-1) f^{1 / 2}}\left[1-\left(1-\frac{f^{1 / 2}}{g_{2}\left(g_{2}^{2-s}-1\right)^{1 / 4}}\right)^{s-1}\right] \\
& \times H\left[f-\left(g_{2}^{2-s}-1\right)^{1 / 2}\right] H\left[g_{2}^{2}\left(g_{2}^{2-s}-1\right)^{1 / 2}-f\right],
\end{aligned}
$$

$$
\begin{aligned}
F_{2}(f)= & (s-1)^{\frac{3-s}{2}} H\left[f-(s-1)^{-1 / 2}\right] \int_{g_{2}^{-1}}^{1} \mathrm{~d} x x^{(2-s)(3-s) / 4} \\
& \times\left[1-(s-1)^{1 / 4} f^{1 / 2} x^{\frac{6-s}{4}}\right]^{s-2} H\left[x-(s-1)^{-\frac{1}{2-s}} f^{-\frac{2}{2-s}}\right] \\
& \times H\left[(s-1)^{-\frac{1}{6-s}} f^{-\frac{2}{6-s}}-x\right]
\end{aligned}
$$

and

$$
\begin{aligned}
F_{3}(f)= & \left(\frac{2-s}{s-1}\right)^{\frac{s-3}{4}} \int_{1}^{\infty} \mathrm{d} x\left[1-\left(\frac{s-1}{2-s}\right)^{1 / 4} f^{1 / 2} x^{5 / 4}\right]^{s-2} \\
& \times x^{\frac{3-s}{4}} H\left[f-\left(\frac{2-s}{s-1}\right)^{1 / 2} \frac{1}{x^{1 / 2}(1+x)^{2}}\right] \\
& \times H\left[\left(\frac{2-s}{s-1}\right)^{1 / 2} \frac{1}{x^{1 / 2}\left(g_{2}^{-1}+x\right)^{2}}\right]
\end{aligned}
$$

respectively. The arguments of the Heaviside function determine the frequency domains where the three terms contribute to the fluence. Note that a nonzero value of the second term (10) occurs for

$(s-1)^{-\frac{1}{2-s}} f^{-\frac{2}{2-s}} \leq x \leq(s-1)^{-\frac{1}{6-s}} f^{-\frac{2}{6-s}}$

which is only possible for frequencies $f \geq(s-1)^{-1 / 2}>1$. 


\subsection{Small frequencies $f \ll(s-1)^{-1 / 2}$}

For the case of small frequencies $f<(s-1)^{-1 / 2}$ the fluence is only determined by the third term (11). The two Heavisidefunctions limit the range of $x$-values to $X_{1} \leq x \leq X_{2}$, with

$X_{1}^{1 / 2}\left(X_{1}+1\right)^{2}=\frac{\left(\frac{2-s}{s-1}\right)^{1 / 2}}{f}$,

$X_{2}^{1 / 2}\left(X_{2}+g_{2}^{-1}\right)^{2}=\frac{\left(\frac{2-s}{s-1}\right)^{1 / 2}}{f}$.

For very low frequencies $f \ll(s-1)^{-1 / 2}$ we find $X_{2}>X_{1} \gg 1$ so that

$X_{1} \simeq \frac{\left(\frac{2-s}{s-1}\right)^{1 / 5}}{f^{2 / 5}}-\frac{4}{5}, X_{2} \simeq \frac{\left(\frac{2-s}{s-1}\right)^{1 / 5}}{f^{2 / 5}}-\frac{4}{5 g_{2}}$.

$F_{3}(f)$ then is very similar to the integral (SL-97) for the steep spectrum case, and we apply approximation (SL-99) to obtain

$F\left(f \ll(s-1)^{-1 / 2}\right) \simeq \frac{4 F_{0}}{5}\left(1-g_{2}^{-1}\right)\left(\frac{2-s}{s-1}\right)^{-1 / 5} f^{-3 / 5}$.

Apart from constants, we obtain the same behaviour as for the steep spectrum that the fluence spectral index equals 0.6 at low frequencies independent of the injected spectral index $s$.

\subsection{High frequencies $f>(s-1)^{-1 / 2}$}

For high frequency values $(f \gg 1)$ the third term (11) does not contribute to the fluence. The first term (9) contributes only at frequencies higher than $f \geq g_{2}^{(2-s) / 2}$. For frequencies $g_{2}^{(2-s) / 2} \leq$ $f \ll g_{2}^{(6-s) / 2}$, much less than the maximum frequency, we obtain

$F_{1}\left(g_{2}^{(2-s) / 2} \leq f \ll g_{2}^{(6-s) / 2}\right) \simeq g_{2}^{-\left(s^{2}-5 s+10\right) / 4}$.

In the second term (10) we substitute $t=(s-1)^{1 / 4} f^{1 / 2} x^{(6-s) / 4}$ to derive

$$
\begin{aligned}
& F_{2}(f \gg 1)=(s-1)^{\frac{3-s}{4}} \int_{g_{2}^{-1}}^{(s-1)^{-\frac{1}{6-s}} f^{-\frac{2}{6-s}}} \mathrm{~d} x x^{(2-s)(3-s) / 4} \\
& \times\left[1-(s-1)^{1 / 4} f^{1 / 2} x^{\frac{6-s}{4}}\right]^{s-2} H\left[x-(s-1)^{-\frac{1}{2-s}} f^{-\frac{2}{2-s}}\right] \\
& =\frac{4}{6-s}(s-1)^{\frac{2-s}{6-s}} f^{-\frac{s^{2}-5 s+10}{2(6-s)}} \int_{(s-1)^{1 / 4} f^{1 / 2} g_{2}^{(s-6) / 4}}^{1} \mathrm{~d} t \\
& \times t^{\frac{s^{2}-4 s+4}{6-s}}(1-t)^{s-2} H\left[t-(s-1)^{-\frac{1}{2-s}} f^{-\frac{2}{2-s}}\right] .
\end{aligned}
$$

Comparing the argument of the Heaviside function with the lower integration limit yields the two frequency ranges with $f_{2}=(s-1)^{-1 / 2} g_{2}^{(2-s) / 2}$

$$
\begin{aligned}
F_{2}(f \gg 1)= & \frac{4}{6-s}(s-1)^{\frac{2-s}{6-s}} f^{-\frac{s^{2}-5 s+10}{2(6-s)}} \\
& \times\left(H\left[f_{2}-f\right] \int_{(s-1)^{-\frac{1}{2-s}} f^{-\frac{2}{2-s}}}^{1} \mathrm{~d} t t^{\frac{s^{2}-4 s+4}{6-s}}(1-t)^{s-2}\right. \\
& \left.+H\left[f-f_{2}\right] \int_{(s-1)^{1 / 4} f^{1 / 2} g_{2}^{(s-6) / 4}}^{1} \mathrm{~d} t t^{\frac{s^{2}-4 s+4}{6-s}}(1-t)^{s-2}\right)
\end{aligned}
$$

which can be expressed in terms of hypergeometric functions allowing no simple analytical form. This implies that a fully analytical solution of the fluence at all frequencies $f \gg 1$ cannot

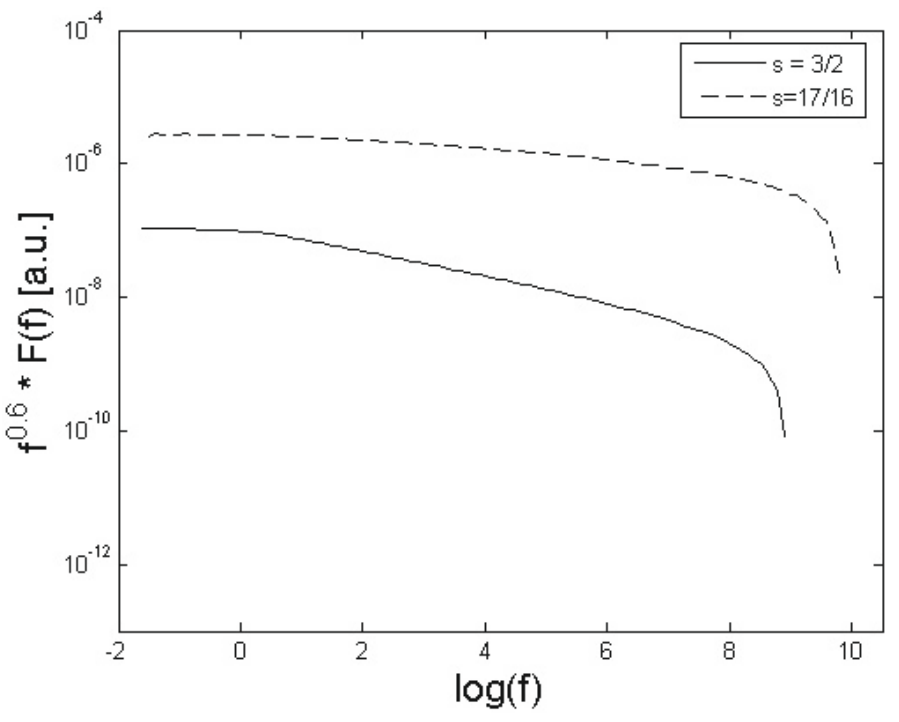

Fig. 1. Nonlinear fluence $F(f)$ in arbitrary units multiplied by $f^{0.6}$ as a function of the normalized frequency $f$ for two values of $s$ for $\gamma_{1}=$ $g_{2}=10^{4}$.

be achieved, so that the integral has to be solved numerically (see Sect. 3).

We note, however, that for frequencies $1 \ll f \ll g_{2}^{(6-s) / 2}$ both lower integration limits are small compared to unity, allowing us to approximate both remaining integrals by the constant

$I_{1}(s)=\int_{0}^{1} \mathrm{~d} t t^{\frac{s^{2}-4 s+4}{6-s}}(1-t)^{s-2}=\frac{\Gamma\left(\frac{s^{2}-5 s+10}{6-s}\right) \Gamma(s-1)}{\Gamma\left(\frac{2(s+2)}{6-s}\right)}$.

Collecting terms we find for the fluence at high frequencies

$$
\begin{aligned}
F\left(1 \ll f \ll g_{2}^{(6-s) / 2}\right) \simeq & \frac{N_{0}}{A_{0} U_{1}^{1 / 2} \gamma_{1}^{s}} f^{-\frac{s+2}{6-s}} \\
& \times\left[\frac{4 I_{1}(s)}{6-s}(s-1)^{\frac{2-s}{6-s}}+\left(\frac{f}{g_{2}^{(6-s) / 2}}\right)^{\frac{s^{2}-5 s+10}{2(6-s)}}\right] \\
\simeq & \frac{N_{0}}{A_{0} U_{1}^{1 / 2} \gamma_{1}^{s}} \frac{4 I_{1}(s)}{6-s}(s-1)^{\frac{2-s}{6-s}} f^{-\frac{s+2}{6-s}} .
\end{aligned}
$$

With respect to the fluence (15) at low frequencies a spectral steepening by

$\Delta(s)=\frac{s+2}{6-s}-\frac{3}{5}=\frac{8(s-1)}{5(6-s)}$

occurs which depends on the value of the injection spectral index $(1<s<2)$. The spectral steepening is limited to values $0<$ $\Delta(s)<(2 / 5)$.

\section{Numerical solution for the fluence}

Because of the problem of the analytical integration for the fluence at high frequencies we calculate the relevant integrals numerically for $\gamma_{1}=10^{4}$ and $g_{2}=10^{4}$. Figure 1 shows the frequency behaviour of the fluence $F(f)$ multiplied by $f^{0.6}$ for the two cases $s=3 / 2$ and $s=17 / 16$. Figure 1 shows a clear break in the fluence spectrum above $f \simeq 1$. The fluence steepens from the $f^{-0.6}$-spectrum to a steeper spectrum. The high-frequency power law spectral index is determined by the injected electron spectral index $s$ in agreement with the analytical steepening (21). 


\section{Discussion}

We have calculated the synchrotron fluence of relativistic electrons for instantaneous injection of a flat power-law spectrum with a spectral index $1<s<2$ and nonlinear synchrotron radiation cooling. At small normalised frequencies the fluence varies proportionally to $\propto f^{-0.6}$ independently of the value of $s$. SL obtained the same behaviour for steep spectral index values $s>2$. At high frequencies $f \gg 1$ the fluence spectrum steepens to a power law determined by the electron injection spectral index $s$.

We conclude that, independent of the steepness of the injected electron spectrum, nonlinear radiative cooling of ultrarelativistic electrons reveals itself by the unique $f^{-0.6}$-synchrotron fluence spectrum at low frequencies which is steeper than the $f^{-0.5}$-synchrotron fluence spectrum at low frequencies for linear cooling (SL). The value of the transition frequency to the unique fluence spectrum depends on the lower cut-off $\gamma_{1}$ of the injected electron power law spectrum.
Acknowledgements. This work was partially supported by the Deutsche Forschungsgemeinschaft through grant Schl 201/16-2.

\section{References}

Aharonian, F. A., Akhperjanian, A. G., Bazer-Bachi, A. R., et al. 2007, ApJ, 664 , L71

Schlickeiser, R. 1996, Space Sci. Rev., 75, 299

Schlickeiser, R., \& Lerche, I. 2007, A\&A, 476, 1

Schlickeiser, R., \& Lerche, I. 2008, A\&A, 485, 315 (SL)

Stawarz, L., \& Petrosian, V. 2008, ApJ, 681, 1725

Stockem, A., Lerche, I., \& Schlickeiser, R. 2007, ApJ, 659, 419

Tammi, J., \& Vainio, R. 2006, A\&A, 460, 23

Vainio, R., \& Schlickeiser, R. 1999, A\&A, 343, 303

Vainio, R., Virtanen, J., \& Schlickeiser, R. 2003, A\&A, 409, 821

Virtanen, J., \& Vainio, R. 2005, ApJ, 621, 313 